Running head: EMOTIONAL CO-REGULATION PROCESSES

\title{
What Can Be Learned From Couple Research: Examining Emotional Co-Regulation Processes in Face-to-Face Interactions
}

\author{
Peter Hilpert \\ University of Surrey \\ Timothy R. Brick \\ Pennsylvania State University \\ Christoph Flueckiger \\ University of Zurich \\ Matthew Vowels \\ University of Surrey \\ Eva Ceuleman and Peter Kuppens \\ University of Leuven \\ Laura Sels \\ University of Zurich
}

\begin{abstract}
Author note
Peter Hilpert, School of Psychology, University of Surrey; Timothy R. Brick, Department of Psychology, Pennsylvania State University; Christoph Flueckiger, Department of Psychology, University of Zurich; Matthew Vowels, School of Psychology, University of Surrey; Eva Ceuleman, Department of Psychology, University of Leuven; Peter Kuppens, Department of Psychology, University of Leuven; Laura Sels, Department of Psychology, University of Zurich.
\end{abstract}


This research was supported by grants from the Swiss National Science Foundation (P3P3P1_174466, P300P1_164582, P2ZHP+_151628).

Correspondence concerning this article should be addressed to Peter Hilpert, School of Psychology, University of Surrey, Guildford, GU2 7AL. E-mail: p.hilpert@ surrey.ac.uk

Hilpert, P., Brick, T., Flueckiger, C., Vowels, M., Kuppens, P., Ceulemans, E., \& Sels, L. (in press). What can be learned from couple research: Examining emotional co-regulation processes in face-to-face interactions. Journal of counselling Psychology. 


\begin{abstract}
A crucial component of successful counseling and psychotherapy is the dyadic emotion coregulation process between patient and therapist which unfolds moment-to-moment during therapy sessions. The major reason for the disappointing progress in understanding this process is the lack of appropriate methods to assess subjectively experienced emotions continuously during therapy sessions without disturbing the natural flow of the interaction. The resulting inability has forced the field to focus on patients' overall emotion ratings at the end of each session with limited predictive value of the dyadic interplay between patient and therapist's emotional states within each session. The current tutorial demonstrates how couple research - confronted with a comparable problem - has overcome this issue by (i) developing a video-based retrospective self-report assessment method for individuals' continuous state emotions without undermining the dyadic interaction and (ii) using a validated statistical tool to analyze the dynamical process during a dyadic interaction. We show how to assess emotion data continuously, and how to unravel self-regulation and co-regulation processes using a Latent Differential Equation Modeling approach. Finally, we discuss how this approach can be applied in counseling psychology and psychotherapy to test basic theoretical assumptions about the co-creation of emotions despite the conceptual differences between couple dyads and therapist-patient dyads. The present method aims to inspire future research activities examining systematic real-time processes between patients and therapists.
\end{abstract}

Keywords: Dynamical systems modelling; Computational psychology; In-session behaviors; Dyadic process; Emotion co-regulation, Latent differential equation modeling

Public Significance Statement. The current tutorial presents a method (i) to assess continuous emotions retrospectively and to examine (ii) dynamic emotion regulation processes 
between patients and therapists during psychotherapy sessions. Such a method gives clients an explicit voice at a moment-to-moment level, allows future research to examine novel research questions, can be used as an educational tool for therapy trainings, and has the potential to enhance our understanding of psychotherapeutic treatments when combined with computational systems that can recognize, interpret, and process human emotions (i.e., affective computing). 


\section{What Can Be Learned From Couple Research: Examining Emotional Co-Regulation Processes in Face-to-Face Interactions}

\section{(a) A Conceptual Introduction To Emotional Co-Regulation}

It is widely accepted that the psychotherapeutic and counseling process relies heavily on social interactions where emotions are an essential part of the dyadic interplay between clients and counsellors (Elliott, 1986; Fosha, 2001; Greenberg, 2015; Hill, 2014; Wampold \& Imel, 2015). Clients and counsellors often explore challenging situations in patients' lives and try to develop new strategies to better cope with such situations. At the same time, however, clients and counsellors experience their own emotions during a session which are co-created on a dyadic moment-tomoment level (e.g., Znoj, Nick \& Grawe, 2004; Frederickson, Messina, \& Grecucci, 2018; Imel et al., 2014; Wiser \& Arnow, 2001). Furthermore, they often encounter difficulties during sessions (i.e., alliance ruptures) which drives them to explore their emotions towards each other (Eubanks-Carter, Muran, \& Safran, 2015).

One of the major reasons for the disappointing progress in understanding the co-creation of emotions during a therapy session is the lack of appropriate self-report methods to assess subjectively experienced emotions continuously during a therapy session without disturbing the natural flow of the interaction. The resulting inability has forced the field to focus on overall sessionevaluations of emotions, allowing for inferences between patients and within patients across sessions (Ulvenes et al., 2014) - but with limited predictive value of quantifying how the dynamic interplay unfolds between two interaction partners during a session (Gottman, Murray, \& Swanson, 2005). The lack of such a self-report method limits our knowledge primarily to overall sessionevaluations and/or to observational data. Thus, a solution is needed to go a step forward to assess how subjectively experienced emotions are co-regulated across client and counsellor at an in-session level (Elliot, 1986). 
Studying the co-creation of emotions is challenging because it involves collecting and analyzing intensive data within a session. Therefore, the aim of the current tutorial is to show how couple research - confronted with a comparable problem - has developed a self-report method to successfully assess emotions continuously. As the resulting coupled time series data is complex, we further demonstrate how the emotional coupling between two people can be analysed.

Incorporating such a method into psychotherapy and counseling research would have several benefits. Essentially it would give clients an explicit voice at a moment-to-moment level - a lasting claim in Counseling Psychology (Elliot, 1986; Kagan \& Schauble, 1969) but also in human interventions generally (Rosen, Brown, Heiman, Leib, 2000; Scholl, Zill, Härter, \& Dirmaier, 2014). Furthermore it would provide a framework to validate already existing observer-ratings to detect emotional stages in the therapy room (e.g., Pascual-Leone, 2018); it would enable investigation of currently untestable basic theoretical assumptions (e.g., accuracy of counsellors; Ickes \& Hodges, 2013; Schmid Mast \& Hall, 2018); it would allow description of co-regulation and re-evaluation of nuances of contemporary theories; providing an understanding of how emotions change in trainees over years of training; and finally, this method allows us to study continuous emotion regulation processes beyond just objective behavior.

\section{(b) How To Assess Emotions Continuously And Analyze Co-Regulation Processes}

Social interaction theories range from more physiological-oriented explanations to broader psychosocial theories (e.g., Baumeister, 2005). As an example for a physiological-oriented theory, the polyvagal theory suggests that the human ability to socially interact with others is based on an underlying biological system (Porges, 2001). Evolutionarily, the central nervous system developed to respond to the social environment in order to maintain physiological homeostasis. Humans constantly evaluate behavioral cues from others during interactions to determine our level of safety or threat. Thus, perceived behavioral cues can perturb a person's biological system and lead to an 
automatic response, where the vagal nerve simultaneously affects a person's emotions and behavioural cues (Porges, 2001).

Extending these assumptions into a dyadic perspective enables us to conceptualize how people influence each other's internal state mutually moment-to-moment during a social interaction: Person A's behavior influences Person B's internal state (e.g., emotions, cognitions) resulting in Person B's behavioral response, which in turn influences Person A's internal state and subsequent response and so on (Bodenmann, 1995). Furthermore, the ability to infer the other person's emotional state based on that person's behavioral cues (e.g., para-verbal) enables humans to calibrate their own behavioral responses continuously to the other person's emotions momentto-moment (Bhatara, Laukka, \& Levitin, 2014).

Although well-established couple theories often conceptualize emotions as an intrapersonal construct, contemporary couple theories go a step further and suggest that emotions can be conceptualized as a Temporal Interpersonal Emotion Systems (TIES; Butler, 2011), simply because emotions serve a social function during an interaction. Imagine, for example, a counsellor-in-training has supported a client intensively - but during a good session, the client accuses the counsellor of not being good enough and wants to discontinue therapy. Currently, such in-session ruptures are usually coded by observers (e.g., Eubanks, Muran, \& Safran, 2018). This example makes it obvious that people influence each other's internal state (e.g., emotions). Emotional co-regulation is a central aspect of a therapeutic interaction and should be conceptualized as an interpersonal system. Thus, the continuous streams of emotional states are interdependent and co-vary during an interaction. On this point of view, a person's emotional state not only depends on the person's selfregulation skills but also is influenced by the other person's emotions and behavior.

In the last decades, dyadic research has focused on several methods to study co-regulation processes in social interactions: retrospective self-report (e.g. surveys, daily diaries, experience sampling, post-session reports), manual observational coding of interactions (either live or video- 
based), and automated coding via artificial intelligence using computer vision tools or wearable physiology monitors. Survey studies often force participants to provide a generalized evaluation across many situations which critically does not capture specific situation nor moment-to-moment processes (e.g., Bodenmann, Hilpert, Nussbeck, \& Bradbury, 2014). Daily diary studies and postsession research allows us to compare effects across days within a person (e.g., Hilpert et al., 2018) and session to session effects ( i.e., lag effects; Atzil-Slonim et al., 2018; Hartmann, Joos, Orlinsky, \& Zeeck, 2015; Kivlighan, Hill, Gelso, \& Baumann, 2016; Zilcha-Mano, Snyder, \& Silberschatz, 2017) but does not allow the study of processes between individuals during a session. Observational coding allows trained coders to rate videotaped behaviors sequentially during a session and there are several observational instruments in the psychotherapy process literature to investigate emotion-related concepts (Dreher, Mengele, Krause, \& Kämmerer, 2001; Eubanks-Carter et al., 2015; Flückiger \& Znoj, 2009). However, external coders can only infer a person's subjectively experienced emotions but never actually know how they feel (Heyman, Lorber, Eddy, \& West, 2014). Finally, there are tools to assess intensive behavioral data (passive smartphone sensors; Weixuan \& McDuff, 2018) and extract facial and vocal signals automatically (Amos, Ludwiczuk, \& Satyanarayanan, 2016; Eyben, Weninger, Gross, \& Schuller, 2013). However, automated tools are in their infancy, and it remains an open question as to how behaviorally displayed emotions correspond to a person's subjectively experienced emotions. Behavioral expression of emotions may be more reflective of social norms (e.g., smiling when greeting someone) and concerns about others' perception (e.g., worrying about therapists' judgement. In sum, all of these methods are only able to approximate the most likely subjective experience of emotions at a moment-to-moment level but may diverge from the actual experience. Thus, we conclude that self-report tools are required to investigate subjectively experienced emotions moment-to-moment.

To overcome these limitations, Gottman used the talk-table procedure where an investigator stopped the interaction after each talk turn, and partners rated their own emotions (Gottman et al., 1976; Markman, 1979). As this approach was highly disruptive, a rating dial was 
later introduced which enabled couples to watch their interaction on video a few days after the interaction and rate their subjective emotions continuously (Gottman \& Levenson, 1985). Nowadays, participants watch the recording directly after the session, and provide a continuous rating of their emotions during the process without interrupting the natural flow of the interaction (Heyman et al., 2014; Reed, Barnard, \& Butler, 2015). Thus, the present system incorporates the continuous and subjective experience of both patient and therapist and allows to model their dynamic interplay.

One further challenge is to analyze the resulting coupled times series data. As emotions oscillate over time, appropriate statistical tools are needed to study how two non-linear systems influence each other mutually. As psychology did not have such advanced modelling techniques in the 1980s (Gottman, Murray, \& Swanson, 2005), researchers were forced to examine aggregated data which in turn undermined the ability to study the co-creation of emotional states over time. As a result, the rating dial approach was used less frequently in couple research over the years until novel statistical methods were introduced to psychology such as e.g., the coupled linear oscillator model (Boker \& Nesselroade, 2002). Thus, the question is whether those methods used in couple research can now be adapted to study co-regulation processes between patient and therapist in therapeutic settings.

What is at stake for psychotherapy and counseling research? Recently, there have been substantial research efforts made to understanding self-report dyadic processes in counseling and psychotherapy research particularly at a post-session report level (e.g., Atzil-Slonim et al., 2015; Hartmann, Joos, Orlinsky, \& Zeeck, 2015; Kivlighan, Hill, Gelso, \& Baumann, 2016; Marmarosh \& Kivlighan, 2012) and observer-based in-session level (Eubanks-Carter et al., 2015). However, the effects of psychotherapeutic interventions unfold as a process between patient and therapist during individual sessions. The absence of a self-report method to capture within session processes may have significant consequences for the field's development. Due to dyadic interplay of emotional cocreation being primarily studied from an observational perspective, we are essentially not able to 
investigate the full scope of understanding the client's subjective experience. Current designs often only allow inferences about differences between patients and dyads or about within-person processes across many sessions (Fisher, Medaglia, \& Jeronimus, 2018) - but not on the unfolding subjectively experienced real-time process within persons and between interaction partners within sessions. The real-time associations between behaviors and the internal states (i.e., emotions, cognitions) for example cannot be explored unless self-report tools assess the internal states continuously. This lack of a self-report moment-to-moment assessment methods becomes increasingly problematic as advancements in assessing behavior automatically (develop through technology) and the field starts drifting into a new area of behaviorism (Grafsgaard, Duran, Randall, Tao, \& D'Mello, 2018). Thus, the goal of the current paper is to introduce a self-report method which allows examination of subjectively experienced emotions continuously even if this new method is not without limitations (e.g., time consuming, retrospective assessment).

\section{c) A Practical Guideline to Assess Emotions Continuously and Analyze Co-Regulation Processes \\ Between Patient and Therapist}

This guideline demonstrates how emotions can be assessed and analyzed successfully for dyadic interactions in general as well as for patient-therapist sessions in particular, by focusing on four aspects: (1) equipment to record dyadic interactions, (2) tools to assess subjective emotions continuously over time, (3) conceptualisation of co-regulation dynamics, and (4) statistical methods to analyze coupled time series data.

\section{1) Video and Audio Equipment}

As discussed above, assessing self-reported emotion continuously is essential to investigating emotional processes within and between persons during an interaction. To assess such data, interactions require video recording and participants subsequently reviewing the footage while rating their emotions continuously using specific software. 
Assessment of video material. Most settings in couple and psychotherapy research use a single HD camcorder to videotape the dyadic interaction from the side so that both persons are visible. Although this is sufficient for people to rate their own emotions, we suggest the use of three cameras. Three cameras allow researchers to capture interactions not just from the side but also a close-up view of each person, conferring two main advantages. It enables researchers to show participants during the emotion rating phase what they see during an interaction -the close-up from the other person. This limits the potential influence on the emotion rating when participants also could observe their own behaviour on video (e.g., facial expressions). Furthermore, this provides new opportunities for inter-disciplinary research (e.g., Grafsgaard et al., 2018). For example, there are new tools to compute facial expression of emotions automatically from video (e.g., OpenFace; Amos et al., 2016) or even extract heart rate signals from close-up videos (e.g., Weixuan \& McDuff, 2018), which greatly expand the analysis opportunities by combining information about participants' internal states (e.g., emotions) and their behaviors.

Assessment of audio material. For practical reasons, couple research and psychotherapy often use the camcorder microphone to record interactions. Although this is sufficient for participants to hear what was said during the interaction and enables them to rate their emotions, we suggest to use a Lavalier microphone for each participant. In line with the suggestion to use multiple video cameras, using two microphones allows separation of the speech signals from the two speakers automatically (a process called diarization; Anguera Miro et al., 2012), which in turn allows the use of open source software (e.g., openSMILE, Praat) for automatic extraction of emotional, semantic, and prosodic information from the audio recording (e.g., speech rate, pause, pitch). This enables researchers to examine the linkage between emotions, vocal signals and facial expressions.

\section{2) Assessing Emotions Continuously Using a Rating Slider}


The video sequence of the patient-therapist interaction is presented to the participants directly at the end of the interaction on a screen. There are several ways for participants to rate their emotions continuously (e.g., knob, slider, joystick) using existing software such as DARMA (Girard \& Wright, 2018) or the software we have developed (Vowels, 2019). Our opensource 'emoTVrater' software (download: https://github.com/matthewvowels1/emotvrater) allows users to rate their own emotions. The software tracks the horizontal position of the computer mouse/trackpad on a frame-by-frame basis as the user rates their emotion when watching the video. Thus, rating a sequence/session will take at least the same time. The benefit of using a mouse/trackpad rather than a joystick, is that joysticks consistently return to a neutral position, potentially contributing bias to a user's rating. A virtual slider is overlaid on the video display so that users have real-time visual feedback as to the current positioning/rating of their emotion on a scale which ranges from negative to positive emotion. The output of the software is a text file that logs the position of the slider and the corresponding video frame number together. The software is continually being updated using the visual software programming language Max/MSP 7. In summary, the emoTVrater open source software allows researchers to capture the continuous self-rated emotions of each participant in a simple and cost-free way.

\section{3) A Conceptual Introduction To Emotion Co-Regulation Processes}

Before one can analyze the continuous emotion data, a conceptual understanding of coupled emotion time series data is necessary. The conceptual understanding will further be useful to define what kind of research questions can be answered with this method. As a starting point, we can simply assume that the emotional states between two persons may co-vary systematically during an interaction. Slightly more complex is the assumption that a person's own current emotional state influences both the person's own subsequent state (i.e., auto-regressive effect) as well as the other person's subsequent state (cross-lagged direct effect; Sels, Ceulemans, Bulteel, \& Kuppens, 2016). Although these are valuable assumptions, they oversimplify the complexity of the 
phenomenon because they do not take into account that the important information is inherent in the oscillatory pattern (e.g., how fast emotions are changing, how far the emotions are from baseline).

Thus, the systems governing emotional states do not only involve direct effects where one person's emotional change influences the subsequent state of the other person (Ross et al., 2017) but rather, a number of forces are acting on their emotional state. Each person's individual regulation process pushes them towards a personal equilibrium - a point that represents their emotional comfort zone, which is statistically the mean over the interaction where the person's emotion fluctuates around (Boker \& Laurenceau, 2006). This regulatory force may be responsive, pressing more strongly away from states far from equilibrium. Further, regulation also attempts to stabilize the individual, acting consistently to slow down change and avoid overreacting or overregulating. In an interactive context, these processes are mirrored by interactions with the other person. That is, the client may respond to the therapist's current affect, and the client may respond to how quickly and in which direction they change.

Due to the complexity of these forces, it can be beneficial to make use of a physical metaphor (Boker \& Laurenceau, 2007). We can imagine the affective state of each person in the dyad as a coiled spring. The further each person's affective state is from their comfort zone, the more their regulatory spring "stretches", and the more strongly their internal emotion regulation pulls them back towards a comfortable affective state. In the dynamical systems literature this is called an equilibrium (e.g., Boker, 2015); in DynAffect (Oravecz \& Brick, 2018) it is referred to as a "home base". During an interaction, the springs for the co-conversants are attached by a third spring (Boker \& Laurenceau, 2006). This third spring is usually weaker than the other two, but pulls on both people, sometimes in the same direction as their internal regulation, and sometimes in opposite directions, resulting in more complicated co-regulatory processes. Like a spring system, this results in oscillations (i.e., mood swings). Periods of levity induced in otherwise very sad conversations as 
the springs bounce from negative affect to positive and back again, for example. This model of regulation as a spring system suggests a process of co-regulation, in which each participant influences each other. In an individual model, a static equilibrium may be a common state, but when two actors exert forces on one another, an ever-changing dynamic balance is more common. This in turn leads to insights into the co-regulation systems at work in interaction, which make up the remainder of this tutorial.

Oscillatory dyadic processes can be best illustrated by observed examples in empirical data (Sels et al., 2019). We inspected the emotions of three couples undergoing a 10 minute conflict interaction, where emotions were assessed on a scale from "very unhappy" to "very happy" (coded as -1 and 1 , respectively), and measured once per second, resulting in 600 data points per person. Figure 1 illustrates the emotions of these three couples. Three things can be observed by cursory examination of these graphs. First, each couple exhibits the up-and-down oscillatory emotional behavior described above. Secondly, the scale and time-course of this oscillation differs between couples. Finally, the way in which partners' emotions covary with each other appears to be quite different across couples. However, inspecting the plots will not help us to answer what seems to be the most pressing question - who is influencing who? (Gottman et al., 2005).

In order to understand the somewhat complicated dynamics of the introduced dyadic system, we first aim to model the regulation system of a single individual. Figure 2 shows a simplified example of one person's emotional state over time. Across a short time window (i.e., 3 data points, or approximately 3 seconds, in our example), we derive three quantities that describe the current state of an individual: level, velocity, and acceleration. The first, level (Figure 2, left panel), is simply the distance between that person's current state and their individual equilibrium. That is, how happy or sad is the person at that specific point in time, relative to their average session level. The second quantity, called velocity (middle panel) measures the rate of change and its direction. That is, how 
fast emotions are changing at a specific moment in time. These two quantities become our predictors in the model.

Acceleration, the last quantity, is our primary outcome. Acceleration is measured by examining the curvature at the current point; it captures the change in velocity over time. In the physical world, acceleration is proportional to force (Newton, 1729). Influences on the acceleration of affect can therefore be thought of as forces that "push" a person's emotional state to be more positive or more negative.

Self-regulation dynamics. The relationships among these three quantities allows us to understand the dynamic characteristics of self-regulation, specifically the relationships between level and acceleration as well as between velocity and acceleration. First, the relationship between (i) level and acceleration describes how strongly my internal regulation processes push to keep me within my affective comfort zone and how strongly they push me to return to it when I am outside of it (Figure 3). A strongly negative relationship here means that as I become sadder, the force towards equilibrium (that is, towards being less sad) pulls more and more strongly. When this force is very strong, a participant will pull quickly back to equilibrium. Any mood swings in a person with a strong recovery force are likely to be rapid. In the metaphor of a spring, this is the strength of the spring itself, pulling rapidly to the centre. As with a spring, this force can sometimes over-pull, resulting in mood swings from positive to negative and back again-a disappointing "down" period following a great positive experience, or an episode of a blissful feeling that follows emergence from a dark period.

The relationship between (ii) velocity and acceleration can be thought of as a dampening force, pushing the participant towards stability. A high negative relationship here means that the faster a person's affect is changing, the stronger the opposing force will be, slowing down changes, and discouraging repeated mood swings (Figure 3). A strong relationship here will eliminate any mood swings, removing the "down" period that might follow a blissful event. By contrast, a 
relationship close to zero indicates a less stable system that may show continued mood swings across a large number of ups and downs.

Co-regulation dynamics. In the context of a dyadic system, the influences across individuals become more intricate, but can still be understood in terms of these same forces. A positive influence of the therapist's level on the client's acceleration means that when the therapist expresses positivity, the force exerted pushes the client in the same direction. Similarly, a positive influence of velocity on acceleration means that as one person cheers up, the other is pushed happier; as one person saddens, the other also is pushed sadder; or in a case of anger, a mutual influence might lead to an escalation. A negative relationship flips the effect-if the therapist expresses negative emotions, the client responds by countering with positive emotion; if they express positivity, the client responds with negative emotionality. Each of these may be adaptive in some interactions-sharing in my co-conversant's joy or sadness may be affiliative, while counterbalancing their emotional state may help to regulate it. Importantly, these forces may not be symmetric between partners, and overlay on each participant's internal regulation, resulting in complicated and elaborate behaviours.

With these various quantities and relationships together, we are able to break apart the different regulatory and co-regulatory forces at work. How strongly does a person regulate their own emotions back to their comfort zone after being pulled away? How much does that event echo, and how quickly does the person stabilize their emotions? In this interaction, how much influence does the other person have on them? To what extent is that influence driven by the person's current state, and to what extent is it driven by the way that they change? Each of these forces can have a strong effect on the overall dynamics of the system.

\section{4) Statistical Modelling Emotion Dynamics Based on Coupled Time Series Data}


There are several methods available to study coupled time series data such as crosscorrelations, cross-lagged models and Coupled Linear Oscillator Models (CLO). Cross-correlation models provide information about synchronous changes in emotion - that is, they describe the situation if emotions across persons covary and it does not matter who influences who. Windowed and time-lagged approaches to cross-correlation (e.g., Brick \& Boker, 2011; Ramseyer \& Tschacher, 2014) provide additional insights into the relationship over time as one time series is displaced in relationship to the other.

Cross-lagged models and vector autoregressive (VAR) models allow us to understand how the state of one person affect the subsequent state of the other person, and can be computed using traditional regression and multilevel modeling (Raudenbush \& Bryk, 2002), structural equation modeling (Newsom, 2015) and dynamic structural equation modeling (e.g., Asparouhov, Hamaker, \& Muthén, 2018). Although it is possible to include a variety of different lags in this type of model or even to model change in continuous time (e.g., Voelkle \& Oud, 2013), these models are first-order systems which model the current affective level for each person in terms of the states of both people at the previous timepoints. These models improve on pure cross-correlation approaches by providing models of both the internal regulation of each individual and the co-regulation between individuals. However, this model neglects the influence of the rate of change in one person on the states and changes in the other. That is, in a VAR model, the effect of a positive expression is the same whether it occurs during a conflict or a positive interaction. In a second-order system (e.g., CLO model), however, the effect of the same incidence can differ in relation to the context.

CLO models expand on these methodologies by providing a model that directly maps influence of not only level of affect, but also rates of change. CLO models conceptualize the system in terms of forces and change. This makes these models excellent choices when we are interested in the processes of change at work in regulation where oscillation (that is, low periods with rebounds 
and high periods with return) and equilibrium maintenance are important, such as self- and coregulation of affect.

To extract the information about the oscillatory patterns, we rely on models from the dynamical systems literature (Boker \& Laurenceau, 2007; Kelso \& Tuler, 1984), which describe types of change in terms of derivatives (e.g. Deboeck, Nicholson, Bergeman, \& Preacher, 2013). Level (formally the zeroth derivatives) refers to the actual measured data-the level of positivity or negativity in emotional expression (see Figure 2). In general, this level is relative to an equilibrium, in this case approximated by the mean over the collected data. If it is likely that the equilibrium drifts over the course of the data set, the data can be detrended to remove a linear trend in order to better approximate the center of equilibrium. Level is written as $x$ in our equations. Velocity (the first derivative) refers to the change in $x$ per unit time and is written $\dot{x}\left(\right.$ i.e., $\left.d x_{(t)} / d t\right)$. Acceleration (the second derivative) refer to the change in velocity per unit and is expressed as $\ddot{x}\left(\right.$ i.e., $\left.d^{2} x_{(t)} / d t^{2}\right)$.

Derivatives are time series measures-that is, they depend not only on the current value of positivity, but also on the preceding and successive states. To approximate them from discrete measurements of level, we use a process called time delay. In time-delay embedding, we duplicate our timeseries several times at a slight lag, such that one row of data might include timepoints 1 to 5 , and the next 2 to 6 , and so on until the end of the timeseries. Because this approach smooths over the effects of dynamic outliers, this embedding improves estimation accuracy (von Oertzen \& Boker, 2010). If the timeseries is sampled very quickly relative to the rate of change, the embedding distance can be changed.

In order to compute the associations between the derivates, we apply a model in the Structural Equation Modeling framework called a Latent Differential Equation (LDE). In this model, the derivatives are considered to be latent factors, and regressed on the measured emotion levels following one-headed arrows. This latent factor structure makes it possible to estimate measurement error while still approximating derivatives precisely. 
The relationship between the latent outcome variable individuals' emotion acceleration (conceptualized in terms of forces) can be modeled as a function of the latent predictors own emotional level and emotion velocity, and those of the partner (co-regulation; co-regulation in bold):

$$
\begin{aligned}
& \ddot{x}=\beta_{1} x+\beta_{2} \dot{x}+\beta_{3} y+\beta_{4} \dot{y}+e_{1(t)} \\
& \ddot{y}=\beta_{5} y+\beta_{6} \dot{y}+\beta_{7} x+\beta_{8} \dot{x}+e_{2(t)}
\end{aligned}
$$

where the client's acceleration $(\ddot{x})$ at time $t$ is predicted by client's level $(\mathrm{x})$, client's velocity $(\dot{x})$ as well as the therapist's level $(y)$ and velocity $(\dot{y})$ at any given point in time. The second equation is conceptualized symmetrically, predicting the therapist's acceleration. A positive parameter $\beta_{3}$ would indicates pressure towards the client (Brick \& Boker, 2011) - when the therapist expresses positive affect, the client's affective state is pushed upwards (and the same, with roles reversed, for $\left.\beta_{7}\right) . \beta_{4}$ indicates the same for velocities - if it is positive, then in the process of expressing more positive states, the therapist pushes the client's affective state upwards as well (and the same, roles reversed, for $\beta_{8}$ ). If negative, each of these has the opposite effect, where the therapist expressing more positivity causes a contrary reaction in which the patient expresses less positivity or more negativity. $e_{(t)}$ indicates the error between the predicted and the actual value, representing other forces on the patient's affect, which might include any number of other characteristics not explicitly modelled here (e.g., fatigue).

\section{d) How To Analyze Emotional Co-Regulation Using Simulated Patient-Therapist Sessions Data}

This tutorial focuses on analyzing dyadic emotion co-regulation based on simulated data. The reason for using simulated data is that the purpose of this article is pedagogical in nature and we wanted to have a data set that could be shared without any worry of participant privacy violation. The tutorial is structured into four steps: method section, data handling, data analysis, and interpretation of results. 


\section{Method section}

Sample. We simulated emotions for 82 patient-therapist interactions. To keep the data, download, and computing time for the analysis simple, we computed 60 data points per person.

\section{Data Handling}

We have separated the data handling in working with raw data and a second part when preparing the data for analyses.

Merging emoTVrater raw data. The emoTVrater produces a file for each participant. Thus, the first goal is to merge all participants' files together and include information of each file name (e.g., dyad ID, session, patient or therapist), potentially aggregate emotion data if the frame-byframe assessment is to detailed (e.g., into one data point per second), and merge the data in a wide format the patient and therapist have an individual column. The R syntax file (emoTVrater_20191114.R) explains each step in detail based on four example data files (e.g., ID.001.t.10.07.2019.txt).

Data Preparation for Analysis. In order to compute the oscillation around the equilibrium correctly, the data needs to be prepared. Data and R syntax can be found in the appendix (TherapyDataSet_2019-07-14.txt, LDE_Model_20191114.R).

Centering and detrending. Variables are centered and detrended. Data is centered by computing and removing each individual's average across the interaction (Boker \& Laurenceau, 2006). Thus, this removes individual differences across people (i.e., some people are happier in comparison to others). Furthermore, data is detrended to remove linear trends. This allows for a better approximation of the equilibrium. Data and R syntax can be found in same appendix mentioned above. 
Time delay embedding. A time delay is needed to compute the derivatives (see Figure 4). This is done by computing lags (see R syntax in LDE_Model_20191114.R). It is important that the window is large enough to capture the phenomenon of interest. Windows with a higher embedding dimension (that is, more lagged points in each row) will be more robust to noisy data. In order to estimate three derivatives (level, velocity, acceleration) and measurement error, at least four timepoints are needed. This approach is important for psychotherapy research as it permits investigation into hypothesis-driven therapist reactions to particular client emotions (e.g., Greenberg, 2015).

Sliding window. Finally, we need to determine what timescale of oscillation we wish to examine. In many cases, the sampling rate is determined by the technology of measurement (e.g. the frame rate of the video) rather than the speed of the process. In order to align the model with the timescale of the process, a delay is chosen, such that the lagged points may have one or more measurements ignored between them (e.g. using measures 1, 3, 5, and 7, or 1, 5, 9, and 13 instead of 1, 2, 3 and 4). A variety of methods for estimating the best delay exist, either from theory (one lag should be close to one-quarter oscillation at best) or using automated methods (Deboeck, Boker, \& Bergeman, 2008). Thus, psychotherapy and counseling researchers have the option to select a delay that targets the specific timescale (e.g. seconds, minutes) of interest. The emoTVrater assesses emotions on a frame-by-frame basis (e.g. 25 frames per second). These frames can then be aggregated if needed (see R syntax in LDE_Model_20191114.R).

\section{Analysis: Computing Self and Co-Regulation Using Latent Differential Equation Modeling}

Figure 5 illustrates the parallel-process LDE model to compute the interdependent data for patients and therapists simultaneously. Statistically, this is similar to a parallel-process quadratic growth curve model where intercept, slope, and quadratic term correspond to level, velocity, and acceleration. The only difference is that the LDE is based on a time delay embedded matrix. To run these models, we have included a labeled R syntax (see LDE_Model_20191114.R). 
We have kept the LDE model as basic as possible since most psychologists are not familiar with coupled oscillatory systems. However, we have included some additional models in the appendix for those who are interested to test (i) how to model an individual patient-therapist dyad, (ii) how to include participants average emotions (i.e., equilibrium) or patients' trait level of depression and test how this influences the self and co-regulation processes during the interaction.

\section{Interpretations of Results}

It is important to note that we have simulated the data and the results we are discussing is simply for didactical reasons - so that the reader gets a better understanding of how LDE results can be interpreted and what kind of questions can be answered. In addition, we are only interpreting the effects between therapist and patient but not between patient and therapist as the interpretation are conceptually the same even if they are theoretically and statistically different (e.g., it might be reasonable to assume that therapists influence patients stronger in general than vice versa).

Although it is also possible to assume that all dyads have equivalent influence and compute a single common model, or to use group iterative processes to define clusters (similar to Gates \& Molenaar, 2012), we computed a model for each dyad ( $N=1$ modeling). This might be especially interesting for therapists and researchers as it allows examination of the forces for each patienttherapist dyad individually. For example, the results of the simulated data for dyad 2 show a significant co-regulating force from therapist on how the patient's emotions move $\left(\beta_{3} y=.28, p<\right.$ .001), indicating that when the therapist expresses a positive or more negative emotional state, the patient tends to begin to express a similar state. Furthermore, the therapist influences how fast the patient's emotion changes $\left(\beta_{4} y=-.39, p=.006\right)$, indicating that when the therapist's emotions become more positive rapidly, the patient resists this change, expressing more negativity instead. A therapist intending to keep the patient positive might then undertake a strategy of maintaining a consistently positive emotional tone, avoiding sudden changes in their emotional tone to avoid that 
reactivity. The syntax to run this model (and the model to examine estimates across all dyads) can be found in LDE_Model_20191114.R.

Like all regression-based models, results are most meaningful to interpret by making comparisons, and designs should reflect this approach. In our example, co-regulating force from the therapist to the patient is weaker for patients with higher levels of depression in comparison to patients with lower depression $(\beta=138.5, p<.001)$. Although these are simulated data, if this were real, it would suggest that patients with more depressive symptoms are more resistant to the regulatory influences of the therapist. This might be due to emotional dampening in the patients themselves (that is, reduced variability overall) or due to changes in perceptual ability in depression. From a therapist's perspective, this would imply that in dealing with more depressed patients, a more vigorous display of emotion should be used when attempting to influence depressed patients' affective states. For an individual patient, a therapist might look to see how sensitive that patient is to the therapist's emotions to titrate how carefully their facial expressions must be controlled.

Thus, there are many possible designs which would allow interpretation of co-regulation meaningfully. For example, one could compare the force within dyads before, during, and after an exposition, or compare the differences in influence as therapeutic alliance builds and is destroyed, or as the patient recovers from depression. In essence, these measures provide a novel means of characterizing differences in the way that therapeutic alliance and intervention manifest across a variety of research or intervention questions.

\section{e) Practical Suggestions}

There are several considerations when implementing this method. For example, the approach was primarily developed using dyadic couple research and attention is needed when translating to interventional research in psychotherapy and counseling. There are conceptual differences between couple dyads and patient-therapist dyads. In Western cultures, partners are 
quite egalitarian whereas patent-therapist dyads do have a mutual arrangement of therapist and patient roles within the therapeutic setting, for example. We assume that these roles will systematically impact the characteristics of the co-regulation process, which are potentially different from couple communications. Along these lines, the assessment of the moment-to-moment dyadic co-regulation of therapists and patients in therapy may help to empirically investigate the dyadic understanding of psychotherapy concepts such as empathic responses, positive reward, allianceruptures, countertransference or exposure interventions based on the continuous self-report assessments in well-specified psychotherapy tasks.

However, assessing emotions continuously is an additional burden for study participants because the post session rating process requires additional time. If rating of the whole interaction is too much in some cases, patients and therapists could rate shorter sequences of their interaction. However, the sequence length completely depends on the task, going from milliseconds (e.g., neurological responses) to seconds (e.g., interruptions) or minutes (e.g. escalation). Even short sequences can be meaningful (e.g., therapist co-regulation within a particular therapeutic task, neurological responses) as long as enough sequences are rated. If the goal is to collaborate with affective science e.g., in respect to cross-validate behavioural algorithms, the continuous assessment emotions during some sequences may already be helpful to verify how reliable behavioral cues are in predicting particular internal states. Along these lines, the present method could help in crossvalidating well-developed observational rating systems targeted for psychotherapy and counseling research (of note that present method requires that video recording and session evaluation are closey related to each other).

In addition, patients might experience some discomfort with video recordings of therapy sessions, limiting the present approach to patients that consent for video recording as well as videobased assessments of in-session emotions. However, a confidential application of videotaping is a standard procedure either for supervision, homework for the student, or for legal safety for patients 
as well as therapists in many psychotherapy institutions (Briggie, Hilsenroth, Conway, Muran, \& Jackson, 2016).

It is an open question as to whether the emotional fluctuations people experience during an interaction is identical to what people report continuously after a session watching their interaction. Thus, the question about 'ground-truth' cannot be fully solved with this method. However, it is reasonable to assume that assessing moment-by-moment is an additional psychological perspective that might provide more detailed information about the trajectories and fluctuations of in-session processes, e.g., in comparison to post-session reports. Furthermore, any assessment of subjective emotions involves some kind of cognitive reflection. Reflecting directly at the end of interaction by watching the interaction on video is maybe the best solution we can currently achieve as assessing emotions during the session without interrupting the therapeutic interaction (which in turn would influence the emotions). Most importantly, this method allows us to eventually study the processes between patient and therapist during the session based on self-report perspectives.

Finally, aligning different data streams assessed with multiple cameras can be challenging. This is less problematic when using well-tested laboratories solutions as all of the videos are fed into a software that aligns with the frames but this is more challenging when using individual camcorders. Simple solutions do exist. The use of a film clapper or a simple two-hand clap visible from all cameras can be a surprisingly precise means to allow alignment of both video and audio should they become desynchronized.

\section{f) Future Directions}

There are three main areas where this method can advance the field. First, this method allows examination of novel research questions which have the potential to advance the field of psychotherapy and counseling research in perspective of dyadic and dynamic real-time processes. Second, this instrument can be used as an educative tool for therapy trainings because trainees can 
verify and improve their predictions of patients' emotions. Finally, in corporation with affective computing and deep learning, it has the potential to enhance our understanding of psychotherapeutic treatments and to improve treatment for patients.

\section{Research}

One of the main aims of this research method is to improve moment-to-moment assessment in respect to self-report perspectives. This method enables the field to examine questions such as self-regulation, co-regulation, and inter-personal real-time processes.

Patient and Therapist's self-regulation. The introduced method allows one to specify novel research questions for patients. For example, are self-regulation process specific / unique to specific moods and emotions (e.g., shame, depressive mood, e. g., Tangney, Stuewig, \& Martinez, 2014)? Are changes in self-regulation skills across sessions associated with mental health improvement?

In addition, this method enables one to ask questions about specific within-therapist effects. In some ways, psychotherapy training can be seen in part as training in self-regulation - to react differently than one would naturally, by putting one's own emotion in the background and patients' problem in front. Such skills often are trained on the side and discussed in supervision and selftherapy (e.g., Geller, Norcross, \& Orlinsky, 2005) but have not been systematically studied at a very basic moment-to-moment level.

Patient and Therapit's co-regulation. The proposed method allows testing of pre-existing theoretical assumptions, how patient and therapist systematically influence each other moment-tomoment. Precise therapists' understanding of patients' emotional states is a typical claim of many psychotherapy theories and the present assessment method might help to deepen our knowledge in respect to therapists' perceptions of clients' emotional stages. As such, this method provides the opportunity to investigate fundamental research questions about the potential fluctuation of emotional stages during sessions. This is particularly interesting as patients may show "silent" 
emotional stages, such as emotional avoidance, dissociations or social desirable masking in particular situations.

Going beyond. We used emotions (i.e., positive-negative valence) as the main example for this tutorial in order to keep it focused. However, this method is not limited to emotional valence. Rather, nearly any construct could be assessed such as a variety of emotions ( e.g., anger, sadness, anxiety; Locke \& Horowitz, 1990). This could lead to a new field of continuous self-rating assessments. Furthermore, therapists could rate patients' emotions continuously, which would allow researchers to examine therapist's empathic accuracy (Ickes \& Hodges, 2013). However, the DARMA software allows rating of two concepts simultaneously (Girard \& Wright, 2018). It is even possible to rate one of eight emotions and its intensity in a circumplex model. This would allow to even include conflicting emotions and motives (e.g., I love and hate this person at the same time). However, rating beyond emotional valence may be difficult to operationalize. Thus, it would be important to develop adequate rating domains for more complex emotions. This could be interesting as patients' emotions are often complex and ambiguous. In addition, the proposed method could be combined with an approach focusing on cognitive moment-to-moment processes (Swift, Tompkins, \& Parkin, 2017). In sum, this method can be broadly applied to explore novel areas beyond emotional valence.

Intra and inter-individual multi-model real-time processes. Psychotherapeutic interactions are complex - they are based on multi-model channels (verbal, non-verbal), intra-individual processes (e.g., emotions, cognition), and inter-individual processes (e.g., verbal, vocal, facial) which unfold in real-time between two interaction partners. The improvement in affective computing allows to capture any behavioral cues automatically. For example, it is possible to assess automatically verbal content (Natural Language Processing; Yang et al., 2019), facial expressions of emotions (e.g., OpenFace), vocal cues (e.g., Praat, OpenSMILE), and even assessing physiological parameters contactless (e.g., heart rate and breathing from video; Weixuan \& McDuff, 2018). In addition, machine learning methods started to operationalize higher order constructs such as 
empathy, potentially allowing to monitor empathy in motivational interviewing in real-time (Xiao, Imel, Georgiou, Atkins, \& Narayanan, 2015). Although such investigations will push psychotherapy and counseling research forward, affective computing inherently comprises a challenge. It can only assess objective behavioral cues - potentially leading us again to an area of, more precise, behaviorism. Therefore, the proposed method becomes increasingly important as it enables the field to incorporate the subjective experience of patients and therapists., we should use the advantages of affective computing by making sure that the fundamental subjective experiences are incorporated into the analyses about multi-modal real-time dynamics. Although an LDE model is limited in how many variables can simultaneously be included, a recent study in couple research demonstrated that deep learning models can be used to compute complex multi-modal dynamics (Grafsgaard et al., 2018).

\section{Therapist Training Implications}

However, this approach has not only the potential to be applied for research purposes but also to train therapists on deliberate practice to accurately detect patients' internal emotional states. Currently, trainees are guessing the internal state of their patients but can hardly verify their assumption. That is, emotional accuracy could be tackled when trainees watch videos of therapy interaction guessing the internal state of the patient and contrasting it with the self-reported emotional experience of the patients. Moreover, therapeutic procedures that are developed to enhance the therapists' understanding of their patients (e.g., plan analysis approach; Caspar, 2007) could be contrasted with trainees that are less educated in such approaches. Along these lines, future research with this method could help to inform trainees and supervisors, giving them a precise understanding and delivered practice of what therapists/trainees may feel during therapy. This could potentially enhance the quality of therapist training, supervision, which may benefit the treatment of patients.

\section{Benefit to Enhance Person-Centered Clinical Research Activities}


There is a lasting claim in evidence-based medicine and interventional research to systematically incorporate patients' point of view into clinical research activities (Miles, 2018), particularly supported by a recent initiative of the World Health Organization. We argue that a selfreport assessment at a moment-by-moment level of patients' internal processes will have an effect on a balanced understanding in the rapidly growing field of the observational assessment of realtime emotions, affective computing, and deep learning approaches. Overall, the more precise selfreport assessment at a moment-by-moment level in combination with affective computing and deep learning methods will influence interventions and how the public perceives mental health interventions. 


\section{References}

Amos, B., Ludwiczuk, B., \& Satyanarayanan, M. (2016). Openface: A general-purpose face recognition library with mobile applications, CMU-CS-16-118, CMU School of Computer Science, Tech. Rep., 2016.

Anguera Miro, X., Bozonnet, S., Evans, N., Fredouille, C., Friedland, G., \& Vinyals, O. (2012). Speaker Diarization: A Review of Recent Research. IEEE Transactions on Audio, Speech, and Language Processing, 20(2), 356-370. https://doi.org/10.1109/TASL.2011.2125954

Asparouhov, T., Hamaker, E. L., \& Muthén, B. (2018). Dynamic Structural Equation Models. Structural Equation Modeling: A Multidisciplinary Journal, 25(3), 359-388. https://doi.org/10.1080/10705511.2017.1406803

Atzil-Slonim, D., Bar-Kalifa, E., Fisher, H., Peri, T., Lutz, W., Rubel, J., \& Rafaeli, E. (2018). Emotional congruence between clients and therapists and its effect on treatment outcome. Journal of Counseling Psychology, 65(1), 51-64. https://doi.org/10.1037/cou0000250

Atzil-Slonim, D., Bar-Kalifa, E., Rafaeli, E., Lutz, W., Rubel, J., Schiefele, A.-K., \& Peri, T. (2015). Therapeutic bond judgments: Congruence and incongruence. Journal of Consulting and Clinical Psychology, 83(4), 773-784. https://doi.org/10.1037/ccp0000015

Baumeister, R. F. (2005). The cultural animal: Human nature, meaning and social life. New York, NY: Oxford University Press.

Bhatara, A., Laukka, P., \& Levitin, D. J. (2014). Expression of emotion in music and vocal communication: Introduction to the research topic. Frontiers in Psychology, 5. https://doi.org/10.3389/fpsyg.2014.00399

Bodenmann, G. (1995). A systemic-transactional conceptualization of stress and coping in couples. Swiss Journal of Psychology, 34-49. 
Bodenmann, G., Hilpert, P., Nussbeck, F. W., \& Bradbury, T. N. (2014). Enhancement of couples' communication and dyadic coping by a self-directed approach: A randomized controlled trial. Journal of Consulting and Clinical Psychology, 82(4), 580-591.

https://doi.org/10.1037/a0036356

Boker, S. M., \& Laurenceau, J. P. (2007). Modeling Contextual Effects in Longitudinal Studies. https://doi.org/10.4324/9780203936825

Boker, Steven M. (2015). Adaptive equilibria: A balancing act of regulation in development. Journal for Person-Oriented Research, 1(1-2), 99-109. https://doi.org/10.17505/jpor.2015.10

Boker, Steven M., \& Laurenceau, J.-P. (2006). Dynamical Systems Modeling: An Application to the Regulation of Intimacy and Disclosure in Marriage. In T. A. Walls \& J. L. Schafer (Eds.), Models for Intensive Longitudinal Data (pp. 195-218). https://doi.org/10.1093/acprof:oso/9780195173444.003.0009

Boker, Steven M., \& Nesselroade, J. R. (2002). A Method for Modeling the Intrinsic Dynamics of Intraindividual Variability: Recovering the Parameters of Simulated Oscillators in Multi-Wave Panel Data. Multivariate Behavioral Research, 37(1), 127-160. https://doi.org/10.1207/S15327906MBR3701_06

Brick, T. R., \& Boker, S. M. (2011). Correlational Methods for Analysis of Dance Movements. Dance Research, 29(supplement), 283-304. https://doi.org/10.3366/drs.2011.0021

Briggie, A. M., Hilsenroth, M. J., Conway, F., Muran, J. C., \& Jackson, J. M. (2016). Patient comfort with audio or video recording of their psychotherapy sessions: Relation to symptomatology, treatment refusal, duration, and outcome. Professional Psychology: Research and Practice, 47(1), 66-76. https://doi.org/10.1037/a0040063 
Butler, E. A. (2011). Temporal Interpersonal Emotion Systems: The "TIES" That Form Relationships. Personality and Social Psychology Review, 15(4), 367-393. https://doi.org/10.1177/1088868311411164

Caspar, F. (2007). Plan Analysis. In In T. Eells (Ed.), Handbook of psycho- therapeutic case formulation (pp. 251-289). New York, NY: Guilford Press.

Deboeck, P. R., Boker, S. M., \& Bergeman, C. S. (2008). Modeling Individual Damped Linear Oscillator Processes with Differential Equations: Using Surrogate Data Analysis to Estimate the Smoothing Parameter. Multivariate Behavioral Research, 43(4), 497-523. https://doi.org/10.1080/00273170802490616

Deboeck, P. R., Nicholson, J. S., Bergeman, C. S., \& Preacher, K. J. (2013). From Modeling Long-Term Growth to Short-Term Fluctuations: Differential Equation Modeling Is the Language of Change. In R. E. Millsap, L. A. van der Ark, D. M. Bolt, \& C. M. Woods (Eds.), New Developments in Quantitative Psychology (Vol. 66, pp. 427-447). https://doi.org/10.1007/978-1-4614-9348-8_28

Dreher, M., Mengele, U., Krause, R., \& Kämmerer, A. (2001). Affective Indicators of the Psychotherapeutic Process: An Empirical Case Study. Psychotherapy Research, 11(1), 99117. https://doi.org/10.1080/713663855

Elliot, R. (1986). Making Children Moral. Educational Theory, 36(3), 289-299. https://doi.org/10.1111/j.1741-5446.1986.00289.x

Eubanks, C. F., Muran, J. C., \& Safran, J. D. (2018). Alliance rupture repair: A meta-analysis. Psychotherapy, 55(4), 508-519. https://doi.org/10.1037/pst0000185

Eubanks-Carter, C., Muran, J. C., \& Safran, J. D. (2015). Alliance-focused training. Psychotherapy, 52(2), 169-173. https://doi.org/10.1037/a0037596 
Eyben, F., Weninger, F., Gross, F., \& Schuller, B. (2013). Recent developments in openSMILE, the munich open-source multimedia feature extractor. Proceedings of the 21st ACM International Conference on Multimedia - MM'13, 835-838.

https://doi.org/10.1145/2502081.2502224

Fisher, A. J., Medaglia, J. D., \& Jeronimus, B. F. (2018). Lack of group-to-individual generalizability is a threat to human subjects research. Proceedings of the National Academy of Sciences, 115(27), E6106-E6115. https://doi.org/10.1073/pnas.1711978115

Flückiger, C., \& Znoj, H. (2009). Zur Funktion der nonverbalen Stimmungsmodulation des Therapeuten für den Therapieprozess und Sitzungserfolg: Eine Pilotstudie. Zeitschrift für Klinische Psychologie und Psychotherapie, 38(1), 4-12. https://doi.org/10.1026/16163443.38.1.4

Frederickson, J. J., Messina, I., \& Grecucci, A. (2018). Dysregulated Anxiety and Dysregulating Defenses: Toward an Emotion Regulation Informed Dynamic Psychotherapy. Frontiers in Psychology, 9. https://doi.org/10.3389/fpsyg.2018.02054

Gates, K. M., \& Molenaar, P. C. M. (2012). Group search algorithm recovers effective connectivity maps for individuals in homogeneous and heterogeneous samples. Neurolmage, 63(1), 310319. https://doi.org/10.1016/j.neuroimage.2012.06.026

Geller, J. D., Norcross, J. C., \& Orlinsky, D. E. (Eds.). (2005). The psychotherapist's own psychotherapy: Patient and clinician perspectives. Oxford ; New York: Oxford University Press.

Girard, J. M., \& C. Wright, A. G. (2018). DARMA: Software for dual axis rating and media annotation. Behavior Research Methods, 50(3), 902-909. https://doi.org/10.3758/s13428-017-0915-5

Gottman, J. M., \& Levenson, R. W. (1985). A valid procedure for obtaining self-report of affect in marital interaction. Journal of Consulting and Clinical Psychology, (53), 151-160. 
Gottman, J. M., Notarius, C., Markman, H., Bank, C., Yoppi, B., \& Rubun, M. E. (1976). J. M., Gottman, Notarius, C, Markman, H., Bank, C, Yoppi, B., \&: Rubin, M. E. (1976). Behavior exchange theory and marital decision making. Journal of Personality and Social Psychology, 34,14-23. Journal of Personality and Social Psychology, (34), 14-23.

Gottman, John M., Murray, J. D., \& Swanson, C. C. (2005). The Mathematics of Marriage: Dynamic Nonlinear Models. Retrieved from https://international.scholarvox.com/catalog/book/88800275?_locale=en

Grafsgaard, J., Duran, N., Randall, A., Tao, C., \& D’Mello, S. (2018). Generative Multimodal Models of Nonverbal Synchrony in Close Relationships. 2018 13th IEEE International Conference on Automatic Face \& Gesture Recognition (FG 2018), 195-202. https://doi.org/10.1109/FG.2018.00037

Greenberg, L. S. (2015). Emotion-focused therapy: Coaching clients to work through their feelings (Second edition). Washington, D.C: American Psychological Association.

Hartmann, A., Joos, A., Orlinsky, D. E., \& Zeeck, A. (2015). Accuracy of therapist perceptions of patients' alliance: Exploring the divergence. Psychotherapy Research, 25(4), 408-419. https://doi.org/10.1080/10503307.2014.927601

Heyman, R. E., Lorber, M. F., Eddy, J. M., \& West, T. V. (2014). Behavioral observation and coding. In In H. T. Reis \& C. M. Judd (Eds.), Handbook of Research Methods in Social and Personality Psychology (2nd ed., pp. 345-372). New York: Cambridge University Press.

Hill, C. E. (2014). Helping skills: Facilitating exploration, insight, and action (Fourth edition). Washington, D.C: American Psychological Association. 
Hilpert, P., Xu, F., Milek, A., Atkins, D. C., Bodenmann, G., \& Bradbury, T. N. (2018). Couples coping with stress: Between-person differences and within-person processes. Journal of Family Psychology, 32(3), 366-374. https://doi.org/10.1037/fam0000380

Ickes, W., \& Hodges, S. D. (2013). Empathic Accuracy in Close Relationships. https://doi.org/10.1093/oxfordhb/9780195398694.013.0016

Imel, Z. E., Barco, J. S., Brown, H. J., Baucom, B. R., Baer, J. S., Kircher, J. C., \& Atkins, D. C. (2014). The association of therapist empathy and synchrony in vocally encoded arousal. Journal of Counseling Psychology, 61(1), 146-153. https://doi.org/10.1037/a0034943

Kagan, N., \& Schauble, P. G. (1969). Affect simulation in interpersonal process recall. Journal of Counseling Psychology, 16(4), 309-313. https://doi.org/10.1037/h0027718

Kelso, J. A. S., \& Tuler, B. (1984). Kelso, J. A. S., \& Tuler, B. (1984). A dynamical basis for action systems. In M. S. Gazzaniga (Ed.), . New York: Plenum.

Kivlighan, D. M., Hill, C. E., Gelso, C. J., \& Baumann, E. (2016). Working alliance, real relationship, session quality, and client improvement in psychodynamic psychotherapy: A longitudinal actor partner interdependence model. Journal of Counseling Psychology, 63(2), 149-161. https://doi.org/10.1037/cou0000134

Locke, K. D., \& Horowitz, L. M. (1990). Satisfaction in interpersonal interactions as a function of similarity in level of dysphoria. Journal of Personality and Social Psychology, 58(5), 823-831. https://doi.org/10.1037/0022-3514.58.5.823

Markman, H. (1979). Application of a behavioral model of marriage in predicting relationship satisfaction of couples planning marriage. Journal of Consulting and Clinical Psychology, 743749.

Marmarosh, C. L., \& Kivlighan, D. M. (2012). Relationships among client and counselor agreement about the working alliance, session evaluations, and change in client symptoms using 
response surface analysis. Journal of Counseling Psychology, 59(3), 352-367.

https://doi.org/10.1037/a0028907

Miles, A. (2018). Evidence-based medicine - 2018. Quo Vadis ? Journal of Evaluation in Clinical Practice, 24(1), 3-6. https://doi.org/10.1111/jep.12924

Newsom, J. T. (2015). Longitudinal structural equation modeling: A comprehensive introduction. New York: Routledge, Taylor and Francis Group.

Newton, I. (1729). Principia.

Oravecz, Z., \& Brick, T. R. (2018). Associations Between Slow- and Fast-Timescale Indicators of Emotional Functioning. Social Psychological and Personality Science, 194855061879712. https://doi.org/10.1177/1948550618797128

Porges, S. W. (2001). The polyvagal theory: Phylogenetic substrates of a social nervous system. International Journal of Psychophysiology, 42(2), 123-146. https://doi.org/10.1016/S01678760(01)00162-3

Ramseyer, F., \& Tschacher, W. (2014). Nonverbal synchrony of head- and body-movement in psychotherapy: Different signals have different associations with outcome. Frontiers in Psychology, 5. https://doi.org/10.3389/fpsyg.2014.00979

Raudenbush, S. W., \& Bryk, A. S. (2002). Hierarchical linear models:Applications and data analysis methods (Vols. 1-1). Thousand Oaks, CA: SAGE Publication.

Reed, R. G., Barnard, K., \& Butler, E. A. (2015). Distinguishing emotional coregulation from codysregulation: An investigation of emotional dynamics and body weight in romantic couples. Emotion, 15(1), 45-60. https://doi.org/10.1037/a0038561

Rosen, C. Brown, J. Heiman, S. Leib, R. (2000). The Female Sexual Function Index (FSFI): A Multidimensional Self-Report Instrument for the Assessment of Female Sexual Function. 
Journal of Sex \& Marital Therapy, 26(2), 191-208.

https://doi.org/10.1080/009262300278597

Ross, J. M., Girard, J. M., Wright, A. G. C., Beeney, J. E., Scott, L. N., Hallquist, M. N., ... Pilkonis, P. A. (2017). Momentary patterns of covariation between specific affects and interpersonal behavior: Linking relationship science and personality assessment. Psychological Assessment, 29(2), 123-134. https://doi.org/10.1037/pas0000338

Schmid Mast, M., \& Hall, J. A. (2018). The Impact of Interpersonal Accuracy on Behavioral Outcomes. Current Directions in Psychological Science, 27(5), 309-314. https://doi.org/10.1177/0963721418758437

Scholl, I., Zill, J. M., Härter, M., \& Dirmaier, J. (2014). An Integrative Model of Patient-Centeredness A Systematic Review and Concept Analysis. PLoS ONE, 9(9), e107828. https://doi.org/10.1371/journal.pone.0107828

Sels, L., Cabrieto, J., Butler, E. A., Reis, H. T., Ceulemans, E., \& Kuppens, P. (2019). The occurrence and correlates of emotional interdependence in romantic relationships. Journal of Personality and Social Psychology.

Sels, Laura, Ceulemans, E., Bulteel, K., \& Kuppens, P. (2016). Emotional Interdependence and WellBeing in Close Relationships. Frontiers in Psychology, 7. https://doi.org/10.3389/fpsyg.2016.00283

Swift, J. K., Tompkins, K. A., \& Parkin, S. R. (2017). Understanding the client's perspective of helpful and hindering events in psychotherapy sessions: A micro-process approach. Journal of Clinical Psychology, 73(11), 1543-1555. https://doi.org/10.1002/jclp.22531 
Tangney, J. P., Stuewig, J., \& Martinez, A. G. (2014). Two Faces of Shame: The Roles of Shame and Guilt in Predicting Recidivism. Psychological Science, 25(3), 799-805. https://doi.org/10.1177/0956797613508790

Ulvenes, P. G., Berggraf, L., Wampold, B. E., Hoffart, A., Stiles, T., \& McCullough, L. (2014). Orienting patient to affect, sense of self, and the activation of affect over the course of psychotherapy with cluster C patients. Journal of Counseling Psychology, 61(3), 315-324. https://doi.org/10.1037/cou0000028

Voelkle, M. C., \& Oud, J. H. L. (2013). Continuous time modelling with individually varying time intervals for oscillating and non-oscillating processes: Continuous time modelling. British Journal of Mathematical and Statistical Psychology, 66(1), 103-126. https://doi.org/10.1111/j.2044-8317.2012.02043.x

von Oertzen, T., \& Boker, S. M. (2010). Time Delay Embedding Increases Estimation Precision of Models of Intraindividual Variability. Psychometrika, 75(1), 158-175. https://doi.org/10.1007/s11336-009-9137-9

Wampold, B. E., \& Imel, Z. E. (2015). The great psychotherapy debate: The evidence for what makes psychotherapy work (Second edition). New York: Routledge.

Weixuan, C., \& McDuff, D. (2018). DeepPhys: Video-Based Physiological Measurement Using Convolutional Attention Networks.

Wiser, S., \& Arnow, B. (2001). Emotional experiencing: To facilitate or regulate? Journal of Clinical Psychology, 57(2), 157-168. https://doi.org/10.1002/1097-4679(200102)57:2<157::AIDJCLP3>3.0.CO;2-8 
Xiao, B., Imel, Z. E., Georgiou, P. G., Atkins, D. C., \& Narayanan, S. S. (2015). "Rate My Therapist": Automated Detection of Empathy in Drug and Alcohol Counseling via Speech and Language Processing. PLOS ONE, 10(12), e0143055. https://doi.org/10.1371/journal.pone.0143055

Yang, Z., Dai, Z., Yang, Y., Carbonell, J., Salakhutdinov, R., \& Le, Q. V. (2019). XLNet: Generalized Autoregressive Pretraining for Language Understanding. ArXiv:1906.08237 [Cs]. Retrieved from http://arxiv.org/abs/1906.08237

Zilcha-Mano, S., Snyder, J., \& Silberschatz, G. (2017). The effect of congruence in patient and therapist alliance on patient's symptomatic levels. Psychotherapy Research, 27(3), 371-380. https://doi.org/10.1080/10503307.2015.1126682 
Table.

Parameter Estimates for Emotion Coupling Processes Between Patient and Therapist Partners Based on Simulated Data For One Dyad (Dyad 2) and Across All Dyads and Across All Dyads

\begin{tabular}{|c|c|c|c|c|c|}
\hline \multirow{2}{*}{\multicolumn{2}{|c|}{ Self-Regulation Pattern }} & \multicolumn{2}{|c|}{ Dyad 2} & \multicolumn{2}{|c|}{ Across All Dyads } \\
\hline & & Est & $p$-value & Est & p-value \\
\hline Level_Patient & $\rightarrow$ Acceleration_Patient & -.49 & $<.001$ & -.19 & $<.001$ \\
\hline Level_Therapist & $\rightarrow$ Acceleration_Therapist &.- .33 & $<.001$ & -.19 & $<.001$ \\
\hline Velocity_Patient & $\rightarrow$ Acceleration_Patient & .24 & .053 & .05 & $<.001$ \\
\hline Velocity_Therapist & $\rightarrow$ Acceleration_therapist & -.02 & .828 & .05 & $<.001$ \\
\hline \multicolumn{6}{|c|}{ Co-Regulation Pattern } \\
\hline Level_Patient & $\rightarrow$ Acceleration_therapist & .12 & $<.001$ & -.01 & .162 \\
\hline Level_Therapist & $\rightarrow$ Acceleration_Patient & .28 & $<.001$ & -.01 & .035 \\
\hline Velocity_Patient & $\rightarrow$ Acceleration_Therapist & .20 & .017 & .02 & .016 \\
\hline Velocity_Therapist & $\rightarrow$ Acceleration_Patient & -.39 & .006 & -.01 & .058 \\
\hline
\end{tabular}

Note. Est $=$ unstandardized estimates; significant coefficients are in bold $(\mathrm{p}<.05$; two-tailed $)$. 


\section{Figure Caption}

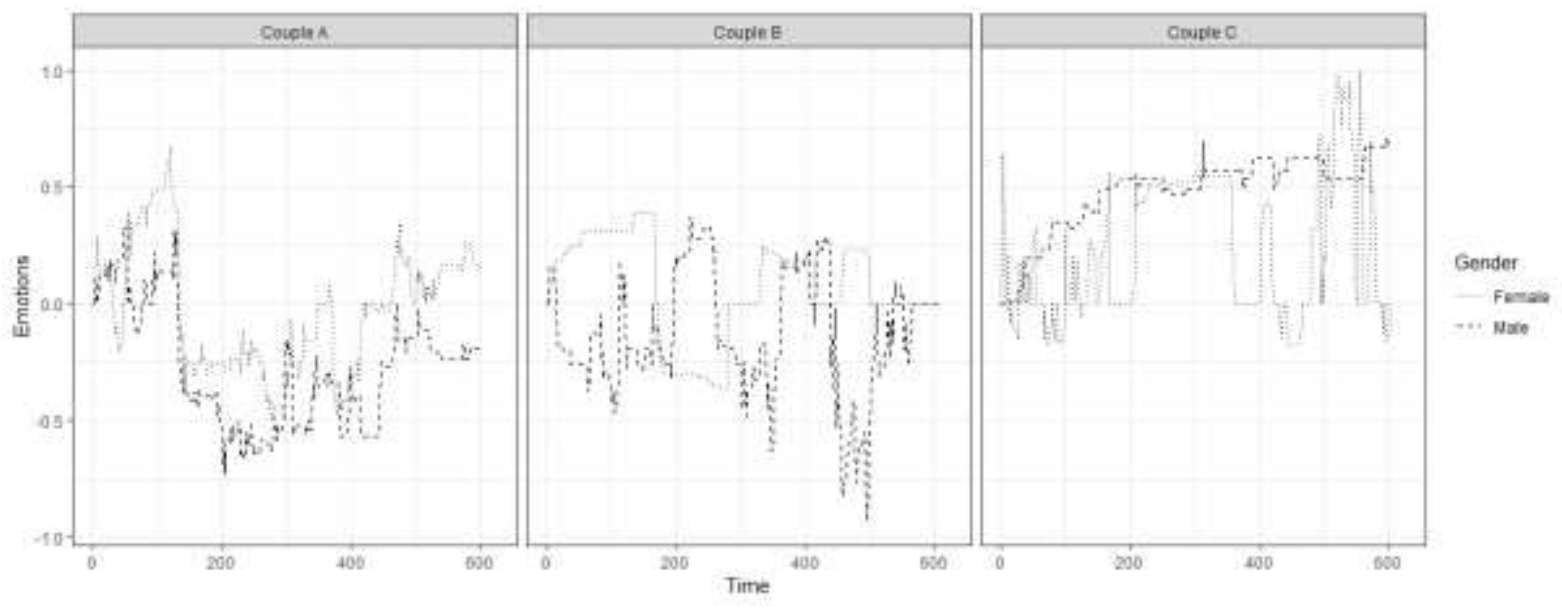

Figure 1. Case study: Emotion variability of three couples within a conflict interaction. 


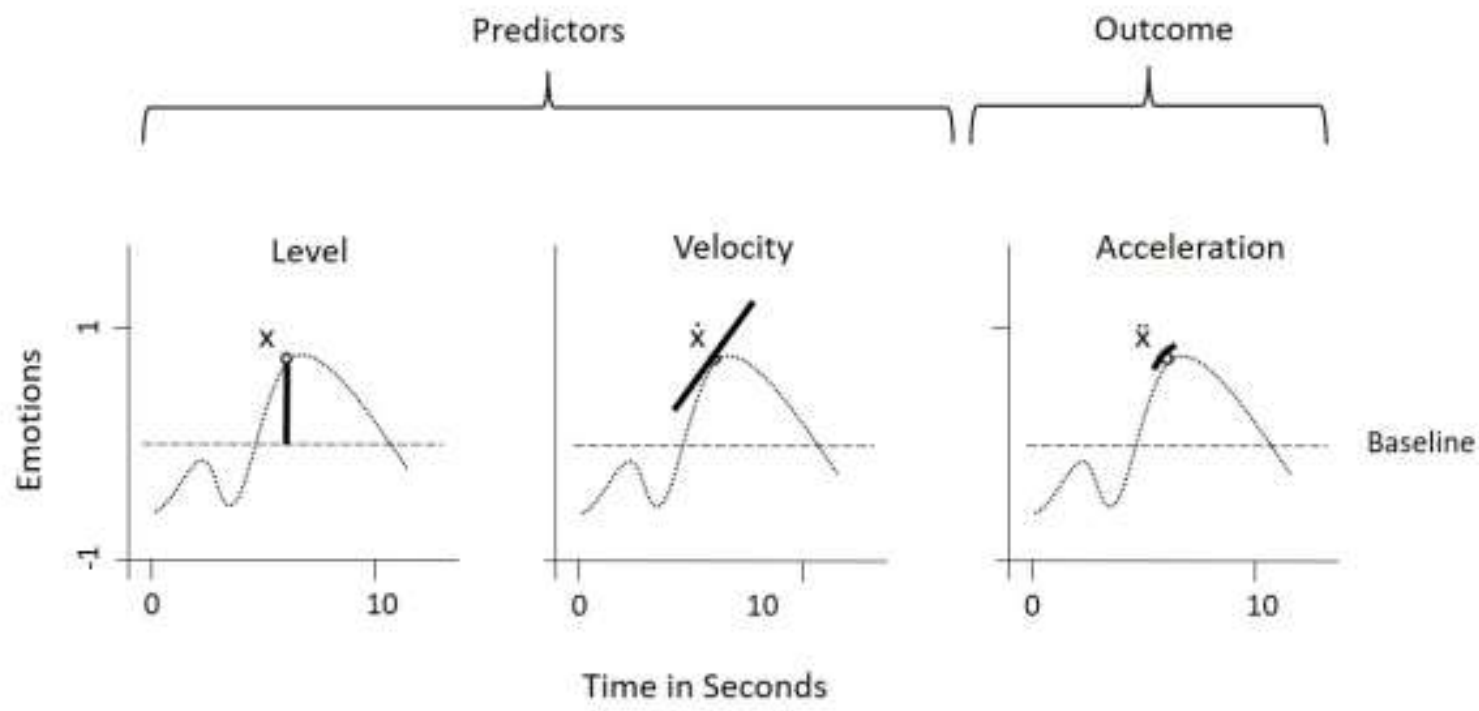

Figure 2. Three quantities derived from an emotional state at a given point in time. Position ( $\mathrm{x}$; left panel) is the value itself, velocity ( $\dot{\mathrm{x}}$; middle panel) is the rate of change, and acceleration ( $\ddot{\mathrm{x}}$; last panel) is curvature or change in velocity. The relationships among these derivatives provide valuable information about the dynamics of the system. 

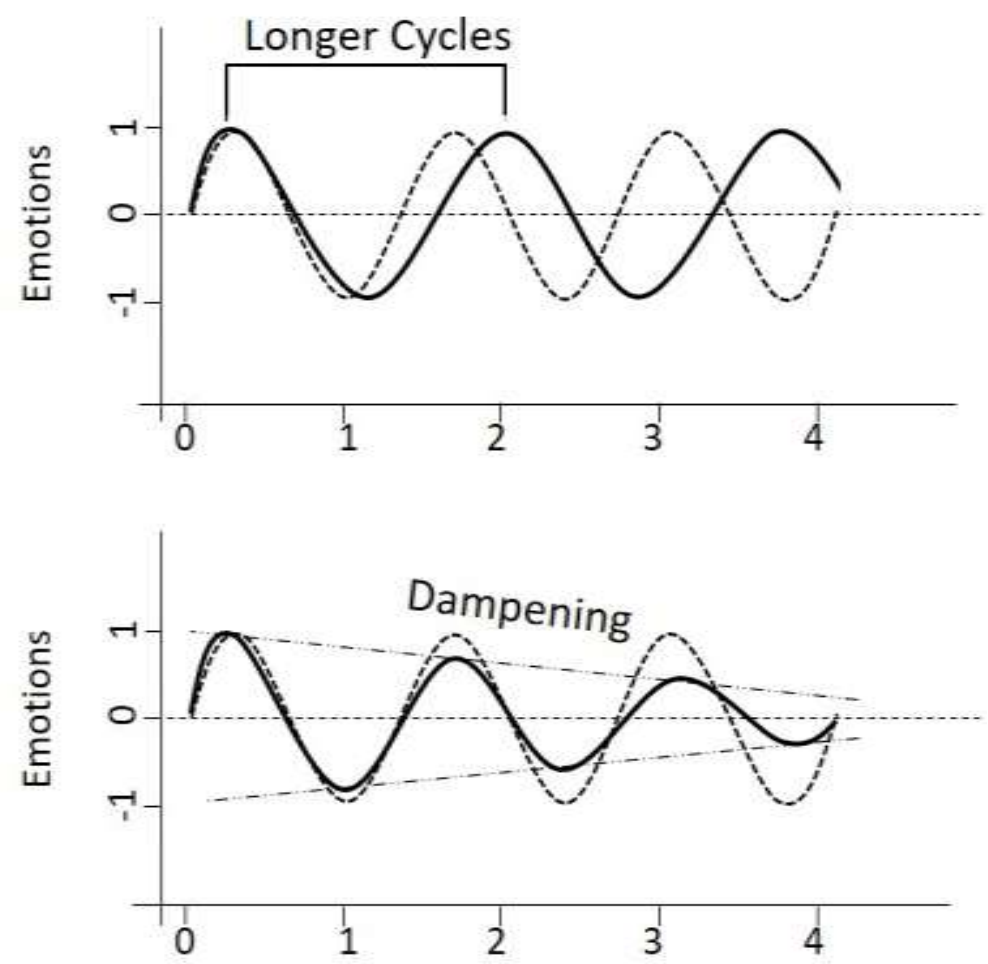

Time

Figure 3. The first panel illustrate the relationship between level and acceleration whereas the second panel illustrates the relationship between velocity and acceleration. 


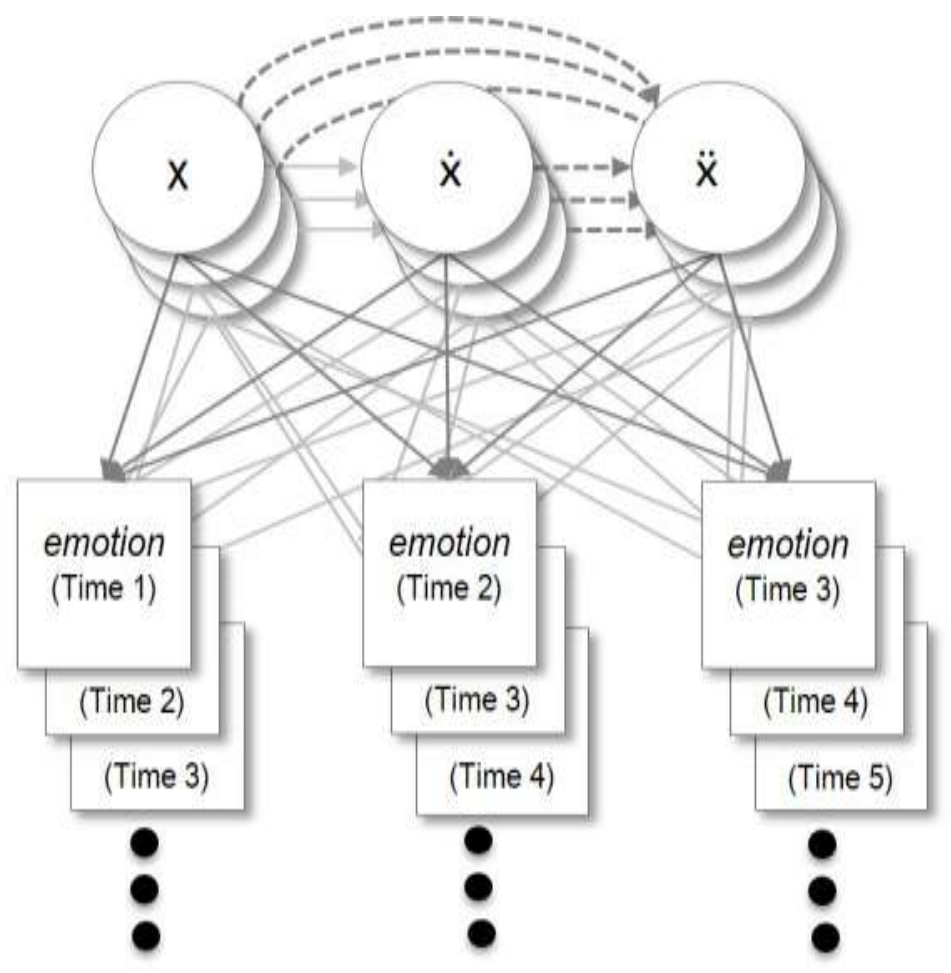

Figure 4. A latent differential equation model based on a time-delay embedded data matrix with a dimension of $3(;)$. Latent derivatives express level/displacement $(x)$, velocity $(\dot{x})$, and acceleration ( $\ddot{x})$ as latent variables. The link between predictors $(x, \dot{x})$ and outcome $(\ddot{x})$ are indicated with dashed lines. 


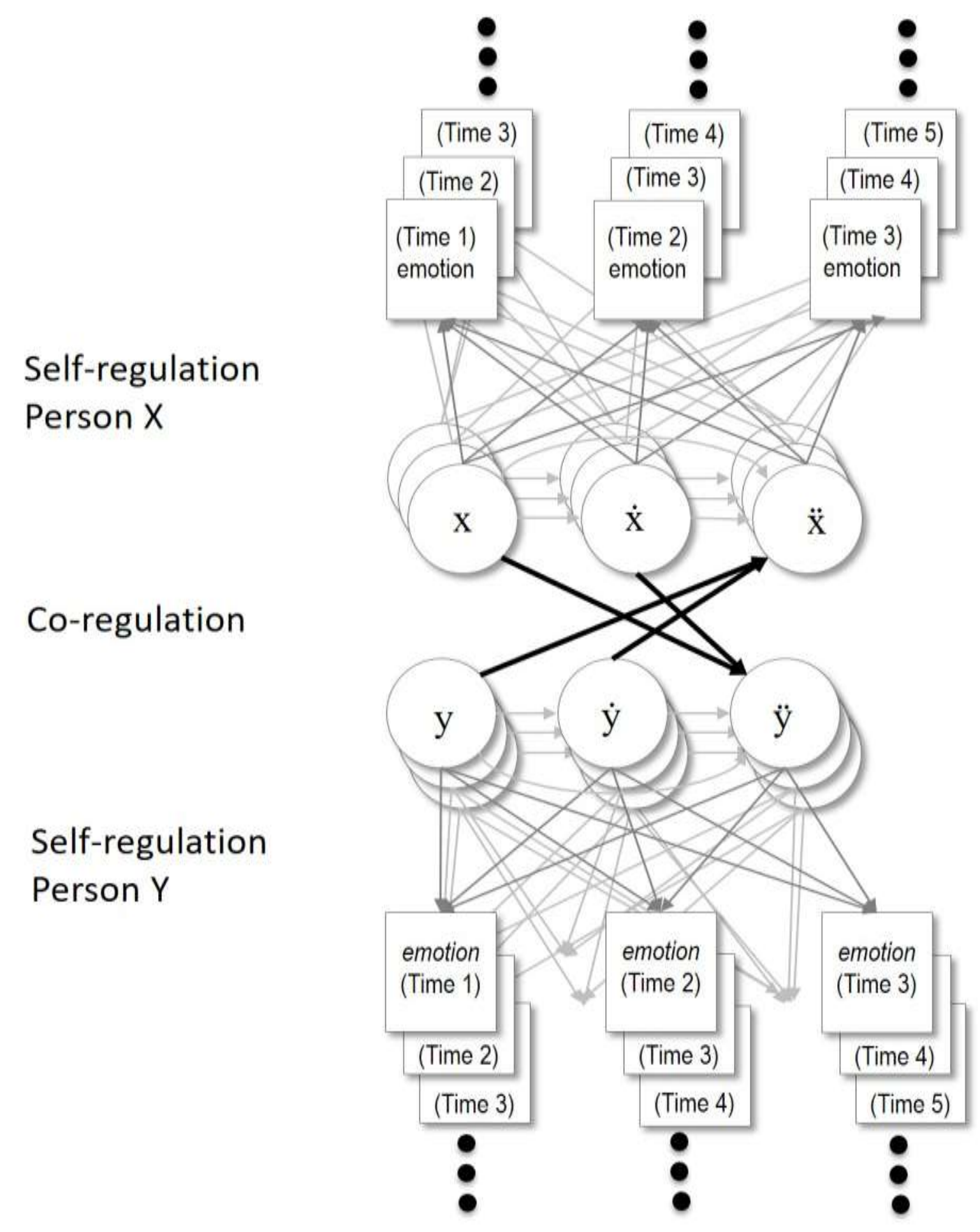

Figure 5. A parallel-process latent derivative equation model, allowing us to compute derivatives and the co-regulation processes between derivatives across the two interaction partners. 